\title{
Les souris mutantes dépourvues d'IRS-2 ont la même résistance périphérique à l'insuline et le même défaut de sécrétion d'insuline que les diabétiques humains de type 2
}

Les diabètes de type 2 (non insulinodépendants) se caractérisent par la coexistence d'une résistance à l'insuline dans les tissus périphériques (muscle, foie, tissu adipeux) et d'une incapacité ou défaillance de la cellule pancréatique $\beta$ à sécréter de façon chronique les quantités relativement abondantes d'insuline qui permettraient de compenser cette résistance $[1,2]$. L'importance relative et l'ordre d'apparition de la résistance à l'insuline et de la déficience sécrétoire sont controversés, mais il semble clair qu'une insulinorésistance n'engendre pas un diabète avéré tant que la cellule $\beta$ reste capable de la compenser. Cette complexité physiopathologique et des arguments d'ordre épidémiologique ont engendré le consensus que la forme commune du diabète de type 2 est une entité hétérogène d'origine multigénique, c'est-à-dire requiert l'action concertée de plusieurs gènes dits diabétogènes, ainsi que de facteurs liés à l'environnement et au style de vie (obésité, manque d'exercice) [1,3]. De fait, malgré de nombreuses recherches, il n'a pas été possible de détecter jusqu'ici chez l'homme de "gène candidat» dont la mutation isolée expliquerait le syndrome diabétique $[4,5]$. Il y a bien des formes familiales monogéniques de diabète comme le MODY (maturity onset diabetes of the young) avec des mutations de la glucokinase $\left(m / s n^{\circ} 6, v o l .8\right.$, p. 600), de facteurs nucléaires hépatiques $\left(\mathrm{m} / \mathrm{s} n^{\circ} 12\right.$, vol. $12, p .1465 ; n^{\circ} 3$, vol. $14, p .364)$ ou de IPF-1 $\left(\mathrm{m} / \mathrm{s} \mathrm{n}^{\circ} 5\right.$, vol. 14, p. 647), ou encore de rares syndromes liés à des mutations, soit de

récepteur insulinique (voir plus loin), soit de protéines mitochondriales [6]. Il s'agit là de phénotypes clairement distincts de la variété commune de diabète de type 2 .

\section{Transmission intracellulaire du signal insuline}

La résistance à l'insuline peut avoir des causes très diverses. Elle peut être, en théorie, due au défaut de n'importe quel élément de la transmission intracellulaire du signal déclenché par l'activation du récepteur membranaire de l'insuline (IR) lors de la liaison de l'hormone [7]. Le premier élément en est l'induction de l'activité tyrosine kinase de IR. La phosphorylation de résidus tyrosine concerne d'abord le récepteur luimême (transphosphorylation), mais aussi toute une série de protéines immédiatement en aval dans la chaîne de transmission intracellulaire. On a découvert il y a une douzaine d'années que le premier chaînon en aval du récepteur, IRS-1 (insulin receptor substrate-1), était une molécule pivot, dépourvue elle-même d'activité enzymatique mais comportant un grand nombre de résidus tyrosine dont la phosphorylation par IR activé induisait la liaison à IRS-1 de molécules effectrices diverses, seconds chaînons de signalisation. On a d'abord cru qu'il y avait là une voie efférente unique et très spécifique du signal insuline, mais le tableau s'est rapidement compliqué. Tout d'abord, il existe d'autres molécules d'ancrage intermédiaires se liant à IR activé. C'est le cas de Shc, dont le rôle dans la chaîne d'activation Ras - MAP- kinase avait été reconnu, ou de Gab1. On a découvert plus récemment que IRS-1 appartient à une famille de protéines dont on connaît à ce jour quatre membres (IRS-1 à -4). Puis, on s'est aperçu que les IRS ne sont pas nécessairement spécifiques de l'insuline (ou des facteurs de croissance semblables à l'insuline, IGF-I et IGFII) mais sont également activés par d'autres stimulus extracellulaires tels que certaines cytokines comme IL-4 ou l'hormone de croissance [7]. Par ailleurs, certains effecteurs sont aussi capables de se lier directement à IR, activant peut-être des voies de signalisation qui court-circuitent les IRS.

\section{Les invalidations géniques}

La création de mutations nulles dans des gènes, chez la souris, a apporté la possibilité de tester expérimentalement le rôle effectif de ces molécules dans l'action de l'insuline [8].

La mutation nulle du récepteur de l'insuline chez la souris entraîne dès la naissance un diabète avec hyperinsulinisme rapidement mortel en quelques jours $[9,10]$. Curieusement, chez l'homme, des mutations du récepteur de l'insuline qui inactivent le récepteur ou en entravent la fonction ou l'expression (y compris quelques cas de mutation nulle des deux allèles) ont été décrites dans plus d'une cinquantaine de cas et ne présentent généralement pas une létalité précoce, mais une résistance sévère à l'insuline associée à des phénotypes divers $\left(\mathrm{m} / \mathrm{s} n^{\circ} 10\right.$, vol. 9 , p. 1139) (léprechaunisme, syndrome d'insulinorésistance avec acanthosis nigricans type A [11], et un cas de 
myopathie congénitale avec disproportion des types de fibres (CFTDM) [12]. Certains patients peuvent survivre jusqu'à l'âge adulte.

On a trouvé chez l'homme quelques polymorphismes dans la séquence d'IRS-1 dans un faible pourcentage de diabètes de type 2 , mais la signification physiopathologique de cette observation reste à établir [13-15]. Dans la mesure où IRS-1 était censé au départ représenter un chaînon aussi essentiel et spécifique dans la transmission du signal insulinique que le récepteur lui-même, on s'attendait à ce que sa mutation nulle chez la souris entraîne également un diabète sévère. En fait, celle-ci n'a eu que des effets relativement mineurs $[16,17]$ : un retard de croissance intra-utérin (expliqué par le fait qu'IRS-1 est aussi un relais du signal du récepteur des IGF), accompagné d'une résistance modérée à l'insuline et d'une hyperinsulinémie compensatrice, avec hypertrophie des cellules $\beta[18]$; la résistance périphérique à l'insuline est essentiellement le fait du muscle, le signal insulinique est quasiment normal dans le tissu hépatique [19]. Ces observations ont été interprétées comme la manifestation d'une redondance fonctionnelle de plusieurs des IRS, l'absence de l'une étant masquée ou compensée par l'activité d'un ou des autres membres de la famille. Cette conviction qu'il existe des situations d'équilibre a été renforcée par une autre observation: alors que chacune des deux mutations nulles de $I R$ et de IRS-1 à l'état hétérozygote est silencieuse [20], on note l'existence d'un diabète sévère chez une souris ayant les deux mutations nulles à l'état hétérozygote. De même, la combinaison chez la souris d'une mutation homozygote d'IRS-1 (entraînant une résistance à l'insuline) et d'un allèle de la glucokinase (entraînant une défaillance dans la sécrétion d'insuline en réponse au glucose) engendre un diabète avéré de type 2 [18]. Ces résultats apportent de l'eau

chez les animaux mutants IRS-2-1-.

Jusqu'à l'âge de quatre semaines, les mutants $I R S-2^{-/-}$ont une sécrétion d'insuline normale en réponse à une charge en glucose, mais cette réponse sécrétoire devient plus modeste quand les animaux sont plus âgés, ce que les auteurs attribuent à une toxicité du glucose due à l'hyperglycémie chronique croissante. Le fait remarquable est que les études morphométriques démontrent une réduc-

au moulin des défenseurs d'une étiologie polygénique du diabète de type 2 .

\section{Invalidation de IRS-2}

C'est dans ce contexte que Whiters $e t$ al. (Boston, MA, USA) [21] révèlent de manière surprenante que l'introduction d'une mutation nulle de IRS2 chez la souris, contrairement à celle de IRS-1, induit à elle seule un diabète de type 2. C'est un diabète qui s'aggrave progressivement, avec acidocétose; les mâles meurent dans un tableau de déshydratation et d'hyperosmolarité après quelques mois, tandis que les femelles, tout en étant atteintes, survivent généralement. Les tests de clamp euglycémique avec injection d'insuline, couplés à l'utilisation de traceurs radioactifs, révèlent une résistance marquée à l'insuline, tant dans le muscle que dans le foie. Les explorations biochimiques $\mathrm{du}$ foie et $\mathrm{du}$ muscle indiquent que le récepteur de l'insuline y est normalement phosphorylé, tandis que l'activité PI3kinase co-immunoprécipitée avec IRS-1 chez les mutants IRS-2-/- est inférieure de $50 \%$ à celle co-précipitée chez l'animal normal. En revanche, l'activité PI3-kinase co-précipitée avec IRS-2 chez les mutants $I R S-1^{-/-}$est plus élevée que celle coprécipitée chez les animaux normaux. Il est donc possible que les défauts de l'activation de la PI3kinase soient responsables de l'anomalie du métabolisme du glucose tion, significative, de plus de la moitié de la masse des cellules $\beta$ dans les îlots pancréatiques des souris IRS-2 $2^{-/}$à l'âge de quatre semaines. Les auteurs signalent avoir retrouvé cette réduction de la masse des cellules $\beta$ dès la naissance, ce qui oriente vers un effet de la mutation indépendant des troubles métaboliques chroniques. L'incrimination de l'absence d'IRS-2 dans les cellules $\beta$ dans le défaut de la réponse insulinique est renforcée par l'observation par immunocytochimie de la co-localisation d'IRS-2 et de l'insuline.

Les souris IRS-2-/- permettent également d'affirmer que des molécules homologues comme IRS-1 et IRS-2 n'ont pas une redondance fonctionnelle absolue. Le phénotype de ces souris réunit les deux caractéristiques essentielles que l'on reconnaît aux diabètes de type 2 chez l'homme : la résistance à l'insuline et une insuffisance de production de l'insuline circulante. A ce titre, ce résultat encourage la recherche active d'anomalies fonctionnelles d'IRS-2 dans les diabètes de type 2 dans l'espèce humaine. Les premiers résultats chez l'homme (une étude portant sur une population de 252 sujets danois) n'ont cependant pas confirmé un rôle génétique pour IRS2 dans le diabète commun de type 2 , aucune association entre les quelques polymorphismes détectés et le diabète n'ayant pu être mise en évidence [22]. Certains facteurs non génétiques ou secondaires à d'autres altérations génétiques pourraient cependant affecter le niveau d'expression d'IRS-2. 
Quelle est la nature du ou des facteurs responsables d'un effet trophique sur la cellule $\beta$ via IRS-2? Il paraît peu probable qu'il s'agisse de l'insuline elle-même, bien que la présence de récepteurs de l'insuline dans la cellule $\beta$ ait été rapportée. Étant donné les concentrations élevées d'insuline dans l'environnement de l'îlot, ces récepteurs seraient probablement saturés et même désensibilisés en permanence, ce qui laisse peu de place à un effet régulateur. Mais le rôle des IGF est à considérer, ainsi que celui de certaines cytokines comme l'hormone de croissance dont l'effet sur les cellules $\beta$ est déjà bien documenté [23]. Ces résultats devraient donner un nouvel élan à l'étude des mécanismes de transmission du signal dans les îlots de Langerhans.

R.L.J.

D.B.

J.J.

P.D.M.

1. Kahn CR. Insulin action, diabetogenes, and the cause of type II diabetes. Diabetes 1994; 43: 1066-84. 2. Lillioja S, Mott DM, Spraul M, Ferraro R, Foley JE, Ravussin E, Knowler WC, Bennett PH, Bogardus C. Insulin resistance and insulin secretory dysfunction as precursors of non-insulin-dependent diabetes mellitus. N Engl J Med 1993 ; 329: 1988-92. 3. De Meyts P. The diabetogenes concept of NIDDM. Adv Exp Med Biol 1993; 334: 89-100.
4. Froguel P. Tracking down genes to cure diabetes; an achievable task for the 21st century? Diabetes Metab 1997; 23 (suppl 2) : 8-13.

5 . Kahn BB. Type 2 diabetes: when insulin secretion fails to compensate for insulin resistance. Cell 1998; 92: 593-6.

6. Froguel P, Vionnet N, Gauguier D, Vaxillaire M, Zouali H, Passa P, Velho G. Génétique du diabète non insulinodépendant. Med Sci 1994; 10 : 795-804.

7. De Meyts P, Christoffersen CT, Tornquist H, Seedorf K. Insulin receptors and insulin action. Curr Opin Endocr Diabet 1996; 3: 369-77.

8. Joshi RL, Jami J. Invalidation chez la souris de gènes susceptibles d'être impliqués dans les diabètes non insulinodépendants. Med Sci 1996; 12: 620-3.

9. Joshi RL, Lamothe B, Cordonnier N, Mesbah K, Monthioux E, Jami J, Bucchini D. Targeted disruption of the insulin receptor gene in the mouse results in neonatal lethality. EMBO J 1996; 15: 1542-7.

10. Accili D, Drago J, Lee EJ, Johnson MD, Cool M, Salvatore P, Asico LD, Jose PA, Taylor SI, Westphal H. Early neonatal death in mice homozygous for a null allele of the insulin receptor gene. Nat Genet 1996; 12: 106-9.

11. Taylor SI, Accili D, Haft CR, Hone J, Imai Y, Levy-Toledano R, Quon MJ, Suzuki Y, Wertheimer E. Mechanisms of hormone resistance: lessons from insulin-resistant patients. Acta Paediatr Suppl 1994; 399: 95-104.

12. Vorwerk P, Vestergaard H, Christoffersen CT, De Meyts P, Pedersen O. Insulin receptor mutation in a novel syndrome of insulin resistance. Diabetologia 1998; 37 (suppl 1) : A29-107 (abstract).

13. Almind C, Bjorbæk C, Vestergaard H, Hansen T, Eschwald S, Pedersen O. Aminoacid polymorphisms of insulin receptor substrate-1 in non-insulin-dependent diabetes mellitus. Lancet 1993; $342: 828-32$.

14. Armstrong M, Haldane F, Taylor R, Alberti
KGMM. Human insulin receptor substrate-1: variant sequences in familial non-insulin-dependent diabetes mellitus. Diabet Med 1996; 13: 133-8. 15. Laakso M, Malkki M, Kekäläinen J, Kuusisto J, Deeb SS. Insulin receptor substrate-1 variants in non-insulin-dependent diabetes. J Clin Invest 1994; 94 : 1141-6.

16. Araki E, Lipes MA, Patti ME, Brüning JC, Haag BI, Johnson RS, Kahn CR. Alternative pathway of insulin signalling in mice with targeted disruption of the IRS-1 gene. Nature 1994; 372: 176-90.

17. Tamemoto H, Kadowaki T, Tobe K, Yagi T, Sakura $\mathrm{H}$, et al. Insulin resistance and growth retardation in mice lacking insulin receptor substrate-1. Nature 1994; 372: 182-6.

18. Terauchi $\mathrm{Y}$, Iwamoto $\mathrm{K}$, Tamemoto $\mathrm{H}$, Komeda K, Ishii C, et al. Development of noninsulin-dependent diabetes mellitus in the double knock-out mice with disruption of insulin receptor substrate-1 and beta-cell glucokinase genes: genetic reconstitution of diabetes as a polygenic disease. J Clin Invest 1997; $99: 861-6$.

19. Yamauchi T, Tobe K, Tamemoto H, Ueki K, Kaburagi $\mathrm{Y}$, et al. Insulin signalling and insulin actions in the muscles and livers of insulin-resistant, insulin receptor substrate 1-deficient mice. Mol Cell Biol 1996; 16: 3074-84.

20. Brüning JC, Winnay J, Bonner-Weir S, Taylor SI, Accili D, Kahn CR. Development of a novel polygenic model of NIDDM in mice heterozygous for IR and IRS-1 null alleles. Cell 1997; 88:561-72. 21. Withers D, Sanchez-Gutierrez J, Towery H, Burks D, Ren JM, Previs S, Zhang Y, Bernal D, Pons S, Shulman G, Bonner-Weir S, White MF. Disruption of IRS-2 causes type 2 diabetes in mice. Nature 1998; 391 : 900-4.

22. Bernal D, Almind K, Yenush L, Ayoub M, Zhang I, Rosshani L, Larsson C, Pedersen O, White MF. IRS-2 amino acid polymorphisms are not associated with random type 2 diabetes among caucasians. Diabetes 1998 (sous presse).

23. Sarvetnik N. Pancreatic growth and regeneration. Basel, Switzerland: Karger Landes Systems, 1997: 250p.

\begin{tabular}{|c|c|c|}
\hline \multicolumn{3}{|c|}{$\begin{array}{l}\text { Deuxième conférence Louis Pasteur sur les maladies infectieuses } \\
\text { SIGNAUX MOLECULAIRES ET MALADIES INFECTIEUSES } \\
\text { 8-10 Octobre } 1998 \bullet \text { Institut Pasteur, Paris, France } \\
\text { La conférence portera sur la pathogénie des maladies infectieuses (parasites, bactéries, virus) } \\
\text { dans le cadre des développements récents en biologie cellulaire. L'accent sera placé sur les voies } \\
\text { de signalisation intracellulaires et les signaux solubles produits par les microbes et leurs hôtes }\end{array}$} \\
\hline \multirow[b]{3}{*}{$\begin{array}{l}\text { Organisateurs } \\
\text { Organizers }\end{array}$} & \multirow[b]{3}{*}{$\begin{array}{l}\text { J.L. Virelizier } \\
\text { (Institut Pasteur, coordinateur) } \\
\text { R.R. Kiberg } \\
\text { K. Joiner } \\
\text { (Yale University) }\end{array}$} & $\begin{array}{l}\text { Conférence inaugurale } \\
\text { Peter C. Doherty (Etats-Unis) }\end{array}$ \\
\hline & & $\begin{array}{l}\text { Signalisation et invasion par les micro-organismes } \\
\text { (adhésion, entrée, fusion, événements précoces) } \\
\text { Norma Andrews (États-Unis), Joan Brugge (États-Unis), Pascale Cossart (France), Jorge E. Calan } \\
\text { (États-Unis), Keith Joiner (États-Unis), Dan Littman (États-Unis), Robert Menard (États-Unis), Phi- } \\
\text { lippe Samsonetti (France), John Skehel (Royaume-Uni). }\end{array}$ \\
\hline & & $\begin{array}{l}\text { Vie et mort des cellules infectées } \\
\text { (immortalisation, apoptose, transmission des signaux, influences réciproques sur la sur- } \\
\text { vie) } \\
\text { Guy Cornelis (Belgique), Michael Donnenberg (États-Unis), Paul Farrell (Royaume-Uni), Alan Hall } \\
\text { (Royaume-Uni), Gordon Langsley (France), Thomas Meyer (Allemagne), David Russel (USA), Jürg } \\
\text { Tschopp (Suisse), Samuel Toreo (États-Unis). }\end{array}$ \\
\hline \multirow{2}{*}{\multicolumn{2}{|c|}{$\begin{array}{l}\text { INSTITUT PASTEUR } \\
\text { Centre d'Information Scientifique } \\
\text { 28, rue du Docteur-Roux } \\
75015 \text { Paris, France }\end{array}$}} & $\begin{array}{l}\text { Signaux solubles } \\
\text { (cytokines, chimiokines, récepteurs solubles, leurres, mécanismes de protection } \\
\text { et d'échappement) } \\
\text { Fernando Arenzana (France), Marco Baggiolini (Suisse), James X. (États-Unis), David Sacks } \\
\text { (États-Unis), Louis Schefield (Australie), Geoffrey Smith (Royaume-Uni) } \\
\text { Présentation des affiches sélectionnées }\end{array}$ \\
\hline & & $\begin{array}{c}\text { Conférences de clôture } \\
\text { Daniel Louvard (France), Stanley Falkow (États-Unis) }\end{array}$ \\
\hline
\end{tabular}

